\title{
A "tail" of opioid receptor variants
}

\author{
Stephanie Puig and Howard B. Gutstein \\ Department of Anesthesiology, University of Pittsburgh, Pittsburgh, Pennsylvania, USA.
}

Opioids are the gold-standard treatment for severe pain. However, potentially life-threatening side effects decrease the safety and effectiveness of these compounds. The addiction liability of these drugs has led to the current epidemic of opioid abuse in the US. Extensive research efforts have focused on trying to dissociate the analgesic properties of opioids from their undesirable side effects. Splice variants of the mu opioid receptor (MOR), which mediates opioid actions, have unique pharmacological properties and anatomic distributions that make them attractive candidates for therapeutic pain relief. In this issue of the $J C l, \mathrm{Xu}$ et al. show that specific C-terminal regions of the MOR can modulate side effects without altering analgesia. This discovery greatly improves our understanding of opioid side effects and suggests intriguing therapeutic approaches that could improve both the safety and long-term effectiveness of opioids.

\section{Background}

Since the time of the ancient Greeks, opioids have been the mainstay of treatment for severe pain (1). The clinical utility of opioids is severely limited by intolerable and potentially life-threatening side effects, such as analgesic tolerance, physical dependence, and addiction liability. Unfortunately, as opioid doses escalate over time to maintain pain relief, tolerance to other potentially devastating side effects, such as respiratory depression, constipation, and itching, do not develop as rapidly. As a consequence, the safety of opioid use decreases markedly, often leaving people suffering from intractable pain.

The analgesic effects of opioids are mediated by the mu opioid receptor (MOR) (2). For over 30 years, investigators have been trying to dissociate the mechanisms that underlie analgesia from those that cause undesirable side effects (3). Several theories to explain opioidinduced side effects, such as tolerance, have been proposed. It was initially sug- gested that tolerance was a consequence of MOR desensitization and internalization, which in turn should lead to the termination of opioid signaling (4); however, the so-called "morphine paradox," in which morphine has been shown to cause profound tolerance without MOR internalization (1), and other data conflict with this hypothesis. It has also been proposed that sustained MOR signaling could cause tolerance (5), but again, there are many data that contradict this suggestion (see ref. 6 for a review). More recently, opioid tolerance and physical dependence were shown to be mediated by specific pathways (7-10). It has long been known that different opioids can induce different profiles of side effects (11). As only one MOR-encoding gene (Oprm1) has been described, it was hypothesized that opioids do not bind to the MOR in a uniform fashion (12). Conformational changes induced by different agonists were thought to distinctly regulate downstream signaling, leading to variations in side-effect profiles. Another

Related Article: p. 1561

Conflict of interest: The authors have declared that no conflict of interest exists.

Reference information: / Clin Invest. 2017;127(4):1221-1224. https://doi.org/10.1172/JCI93582. 
$\mathbf{A}$

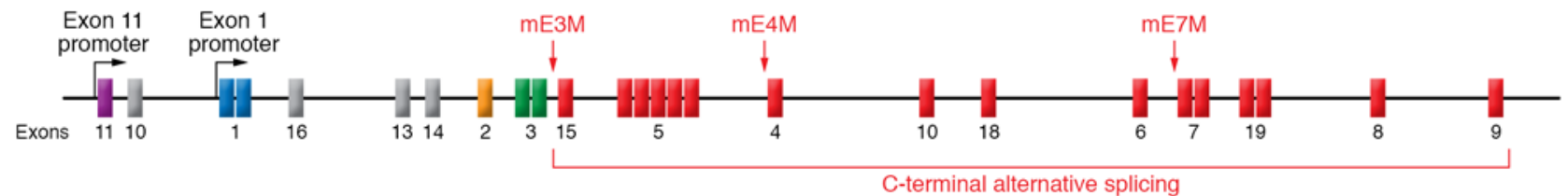

C-terminal alternative splicing

B

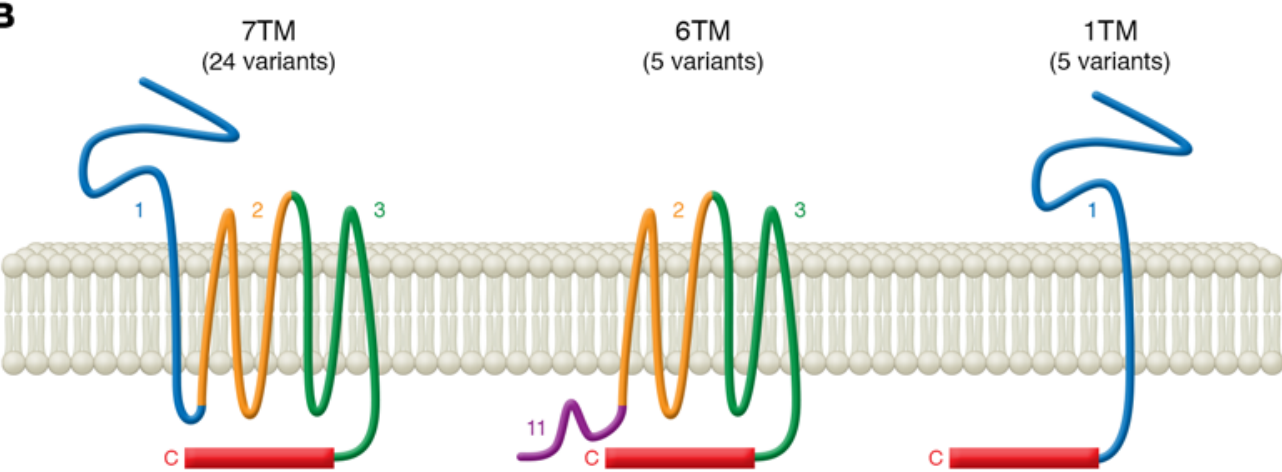

Figure 1. Mouse MOR (Oprm1) gene schematic structure and alternative splicing. (A) Oprm1 gene structure. Exons are indicated by color-coded boxes. Exons 1 (blue), 2 (yellow), and 3 (green) constitute the TM domains of the receptor. The mouse Oprm1 gene contains ten alternatively spliced exons (red boxes) downstream of exon 3 that generate different C-terminal tails. In this issue, Xu et al. report on their generation of mouse models (mE3M, mE4M, and $\mathrm{mE7M}$ ) that express alternatively spliced variants with different $C$ termini (25). Arrows indicate the location of the STOP codons that were inserted to generate each truncation. Consequences of specific C-terminal truncations on opioid-induced side effects are summarized in Table 1. (B) The three classes of MOR splice variants include the classical 7TM form as well as 6TM and 1TM versions. Color-coded structures match the color-coded exons in A. The red rectangles represent the alternatively spliced C-terminal tails of the receptor variants.

significance of these variants has not been explored. In this issue, Xu et al. (25) address this important point by generating 3 different congenic strains of mice on two genetic backgrounds (C57/BL6 and Sv129 mice) that are known to have different behavioral responses to opioids. Each congenic strain had a different truncation in the C-terminal tail of the MOR. The exon $3(\mathrm{mE} 3 \mathrm{M})$ truncation completely eliminated the intracellular C-terminal tail of the MOR, while the $\mathrm{mE} 4 \mathrm{M}$ and $\mathrm{mE7M}$ truncations eliminated the expression of variants that encoded exon 4 and exon 7, respectively.
The $\mathrm{mE} 3 \mathrm{M}$ and $\mathrm{mE} 4 \mathrm{M}$ truncations, but not the $\mathrm{mE7M}$ mutation, reduced receptor binding and caused a similar reduction in GTP $\gamma$ s activation, suggesting that $\mathrm{C}$-terminal truncations of the MOR do not alter opioid-induced $G$ protein coupling. Interestingly, only the complete C-terminal truncation $(\mathrm{mE} 3 \mathrm{M})$ altered the acute analgesic effect of opioids, causing a slight increase in the $\mathrm{ED}_{50}$ of morphine. $\mathrm{Xu}$ et al. then went on to establish an important role for specific C-terminal regions in mediating opioid side effects (Table 1). Remarkably, they observed that the $\mathrm{mE} 3 \mathrm{M}$ and $\mathrm{mE} 4 \mathrm{M}$ truncations enhanced morphine tolerance and reduced physical dependence, but had no effect upon the rewarding properties of morphine. In contrast, the $\mathrm{mE7M}$ truncation reduced tolerance and the rewarding properties of morphine, but had no effect upon physical dependence. Other side effects were also affected, with $\mathrm{mE} 4 \mathrm{M}$ and $\mathrm{mE} 7 \mathrm{M}$ mice showing decreases in locomotor activity and $\mathrm{mE} 4 \mathrm{M}$ mice showing less inhibition of gastrointestinal (GI) transit. All of the C-terminal mutants caused a partial reduction in

Table 1. Summary of the impact of mutations mE3M, mE4M, and mE7M on opioid-induced side effects

\begin{tabular}{|c|c|c|c|}
\hline & mE3M (truncation of all C termini) & mE4M (truncation of exon 4-encoding C termini) & mE7M (truncation of exon 7-encoding C termini) \\
\hline Acute analgesia & Slight decrease & No change & No change \\
\hline Locomotor activity & No change & Reduced & Reduced \\
\hline Tolerance & Enhanced & Enhanced & Reduced \\
\hline Physical dependence & Reduced & Reduced & No change \\
\hline Reward & No change & No change & Reduced \\
\hline Inhibition of GI transit & No change & Reduced & No change \\
\hline Catalepsy & Reduced & Reduced & Reduced \\
\hline
\end{tabular}

Bold text denotes opioid effects that were reduced by mutation, italics represent actions that were enhanced by mutation, and regular text indicates actions that were overall unchanged by the indicated mutation. 
morphine-induced catalepsy, suggesting that the entire MOR C-terminus could play a role in mediating this effect.

As mentioned above, desensitization of MOR-G protein coupling has been postulated to underlie opioid tolerance. $\mathrm{Xu}$ and colleagues used GTP $\gamma$ S stimulation as a measure of coupling efficiency in brain regions of WT and $\mathrm{mE7M}$ mice chronically treated with morphine to determine whether this mutation would block tolerance-induced decreases in GTP $\gamma S$ activation. The authors discovered that the $\mathrm{mE7M}$ mutation blocked morphineinduced decreases in GTP $\gamma \mathrm{S}$ stimulation in the hypothalamus and brain stem, confirming previous studies suggesting that these regions could be important neural substrates of morphine tolerance (26).

An important concept in GPCR signaling is that of biased agonism. This refers to the observation that agonists for GPCRs can preferentially mediate the induction of specific downstream signals. Perhaps the best-studied example of biased agonism is the distinction between stimulation of $G$ protein-mediated signals and $\beta$-arrestin-mediated signaling downstream of GPCRs (13). In the case of opioid receptors, $\beta$-arrestin-mediated signaling is thought to mediate tolerance (8). To determine whether these mutations were biased toward $G$ protein or $\beta$-arrestin 2 signaling, Xu et al. compared signaling bias of various opioids in two $\mathrm{mE7M}$-associated 7TM opioid receptor variants, the mMOR$1 \mathrm{C}$ and mMOR-1O, with the $\mathrm{mE} 4 \mathrm{M}$-associated mMOR-1. They discovered that all tested ligands had a greater bias toward stimulating $\beta$-arrestin 2 in the $\mathrm{mE7M}$-associated variants mMOR-1C and mMOR-1O. This observation is consistent with previous findings demonstrating that $\beta$-arrestin 2 plays an important role in mediating opioid tolerance (8). Interestingly, the phenotype of $\mathrm{mE7M}$ animals was similar to that of $\beta$-arrestin 2 knockout mice, with both strains demonstrating reduced morphine tolerance, loss of receptor desensitization in the brain stem, and no effect upon physical dependence (8).

\section{Therapeutic implications and concluding remarks}

This paper by $\mathrm{Xu}$ and colleagues (25) represents a major advance in our understanding of MOR signaling and the poten- tial therapeutic approaches implied by the diversity of these receptors. First, the authors establish that the extensive $3^{\prime}$ alternative splicing of the MOR has physiologic and functional significance. They show that different mutations of the C-terminal tail can profoundly alter opioid-induced side effects without affecting the analgesic properties of opioids. This study adds additional support to the growing body of evidence that the desirable analgesic effects of opioids can be dissociated from undesirable side effects. This work also demonstrates that side effects can be uncoupled at the level of specific C-terminal regions of the MOR and implies that selectively targeting specific regions of the MOR C terminus could be an effective therapeutic strategy for the reduction of opioid side effects. Xu and colleagues provide proof of this concept by targeting exon 7 of the MOR in WT mice with antisense technology. This treatment markedly reduced morphine tolerance, further supporting the role of exon 7 variants in tolerance and validating this region as a novel therapeutic target. A recent study utilizing high-throughput combinatorial structurebased computational biology bioinformatics and pharmacology to screen thousands of compounds in silico and predict effects based on their MOR docking properties (27) could represent a powerful approach to developing drugs targeting the exon 7-encoding MOR splice variants and ultimately reducing side effects. $\mathrm{Xu}$ and colleagues' observation that the $\mathrm{mE7M}$ mutation decreased the rewarding properties of opioids is also extremely important. This suggests that there could be a mechanistic link between opioid tolerance, reward, and ultimately addiction. While this concept is highly speculative, it is conceivable that therapies targeting exon 7-encoding MOR splice variants could decrease the abuse and addiction liability of opioids. Future studies could have important implications not only for pain treatment, but also for the current epidemic of opioid abuse that is sweeping the US.

In conclusion, this study by $\mathrm{Xu}$ and colleagues (25) establishes the importance of considering the diversity of MOR alternative splice variants and their distinct signaling responses to our understanding of the mechanisms that underlie opioidinduced side effects. The work constitutes a major advance in our understanding of the very complex relationship between MOR signaling and the physiologic effects of opioids.

\section{Acknowledgments}

Work in the Gutstein lab is supported by grant no. 1R01DA036680 from the NIH National Institute on Drug Abuse.

Address correspondence to: Howard B. Gutstein, 3550 Terrace Street, Scaife Hall, Room A-1307, Pittsburgh, Pennsylvania 15261, USA. Phone: 412.647.2813; E-mail: gutsteinhb@upmc.edu.

1. Gutstein HB, Akil H. Opioid analgesics. In: Hardman JG, Limbird LE, eds. Goodman And Gilman's The Pharmacological Basis Of Therapeutics. 10th ed. 2001:569-619.

2. Matthes HW, et al. Loss of morphine-induced analgesia, reward effect and withdrawal symptoms in mice lacking the mu-opioid-receptor gene. Nature. 1996;383(6603):819-823.

3. Ling GS, MacLeod JM, Lee S, Lockhart SH, Pasternak GW. Separation of morphine analgesia from physical dependence. Science. 1984;226(4673):462-464.

4. Ferguson SS. Evolving concepts in G protein-coupled receptor endocytosis: the role in receptor desensitization and signaling. Pharmacol Rev. 2001;53(1):1-24.

5. He L, Fong J, von Zastrow M, Whistler JL. Regulation of opioid receptor trafficking and morphine tolerance by receptor oligomerization. Cell. 2002;108(2):271-282.

6. Williams JT, et al. Regulation of $\mu$-opioid receptors: desensitization, phosphorylation, internalization, and tolerance. Pharmacol Rev. 2013;65(1):223-254.

7. Bohn LM, Gainetdinov RR, Lin FT, Lefkowitz RJ, Caron MG. $\mu$-Opioid receptor desensitization by $\beta$-arrestin-2 determines morphine tolerance but not dependence. Nature. 2000;408(6813):720-723.

8. Burma NE, et al. Blocking microglial pannexin-1 channels alleviates morphine withdrawal in rodents [published online ahead of print January 30, 2017]. Nat Med.

https://doi.org/10.1038/nm.4281.

9. Corder $\mathrm{G}$, et al. Loss of $\mu$ opioid receptor signaling in nociceptors, but not microglia, abrogates morphine tolerance without disrupting analgesia. Nat Med. 2017;23(2):164-173.

10. Wang Y, Barker K, Shi S, Diaz M, Mo B, Gutstein HB. Blockade of PDGFR- $\beta$ activation eliminates morphine analgesic tolerance. Nat Med. 2012;18(3):385-387.

11. Kuo A, Wyse BD, Meutermans W, Smith MT. In vivo profiling of seven common opioids for antinociception, constipation and respiratory depression: no two opioids have the same profile. Br J Pharmacol. 2015;172(2):532-548.

12. Piñeyro G, Archer-Lahlou E. Ligand-specific receptor states: implications for opiate receptor signalling and regulation. Cell Signal. 
2007;19(1):8-19.

13. Markovic D, Challiss RA. Alternative splicing of $\mathrm{G}$ protein-coupled receptors: physiology and pathophysiology. Cell Mol Life Sci. 2009;66(20):3337-3352.

14. Pasternak GW, Childers SR, Snyder SH. Opiate analgesia: evidence for mediation by a subpopulation of opiate receptors. Science. 1980;208(4443):514-516.

15. Pan YX. Diversity and complexity of the mu opioid receptor gene: alternative pre-mRNA splicing and promoters. DNA Cell Biol. 2005;24(11):736-750.

16. Pasternak GW, Pan YX. Mu opioids and their receptors: evolution of a concept. Pharmacol Rev. 2013;65(4):1257-1317.

17. Xu J, et al. Differential expressions of the alternatively spliced variant mRNAs of the $\mu$ opioid receptor gene, OPRM1, in brain regions of four inbred mouse strains. PLoS One. 2014;9(10):e111267.
18. Bolan EA, Pan YX, Pasternak GW. Functional analysis of MOR-1 splice variants of the mouse $\mu$ opioid receptor gene Oprm. Synapse. 2004;51(1):11-18.

19. Shabalina SA, et al. Expansion of the human $\mu$-opioid receptor gene architecture: novel functional variants. Hum Mol Genet. 2009;18(6):1037-1051.

20. Convertino M, et al. Differential regulation of 6and 7-transmembrane helix variants of $\mu$-opioid receptor in response to morphine stimulation. PLoS One. 2015;10(11):e0142826.

21. Pan YX, Xu J, Xu M, Rossi GC, Matulonis JE, Pasternak GW. Involvement of exon 11-associated variants of the mu opioid receptor MOR-1 in heroin, but not morphine, actions. Proc Natl Acad Sci US A. 2009;106(12):4917-4922.

22. Marrone GF, et al. Truncated $\mu$ opioid GPCR variant involvement in opioid-dependent and opioid-independent pain modulatory systems within the CNS. Proc Natl Acad Sci U S A.
2016;113(13):3663-3668.

23. Xu J, et al. Stabilization of the $\mu$-opioid receptor by truncated single transmembrane splice variants through a chaperone-like action. J Biol Chem. 2013;288(29):21211-21227.

24. Tanowitz M, Hislop JN, von Zastrow M. Alternative splicing determines the post-endocytic sorting fate of G-protein-coupled receptors. J Biol Chem. 2008;283(51):35614-35621.

25 . Xu J, et al. Alternatively spliced mu opioid receptor C termini impact the diverse actions of morphine. JClin Invest. 2017;127(4):1561-1573.

26. Sim-Selley LJ, et al. Region-dependent attenuation of mu opioid receptor-mediated G-protein activation in mouse CNS as a function of morphine tolerance. Br J Pharmacol. 2007;151(8):1324-1333.

27. Manglik A, et al. Structure-based discovery of opioid analgesics with reduced side effects. Nature. 2016;537(7619):185-190. 